\title{
IUE TEMPERATURES FOR WHITE DWARF STARS IN AND AROUND THE ZZ CETI INSTABILITY STRIP
}

\author{
S. O. KEPLER \\ Instituto de Física, Universidade Federal do Rio Grande do Sul, 91500 Porto Alegre, RS, Brazil and Département de Physique, \\ Université de Montréal, Montréal, PQ H3C 3J7, Canada \\ E. P. Nelan \\ Space Telescope Science Institute, Homewood Campus, 3700 San Martin Drive, Baltimore, Maryland 21218 \\ Received 29 August 1991; revised 5 October 1992
}

\begin{abstract}
We have analyzed all the archival IUE images of the DA4 and DA5 white dwarfs with model atmosphere fluxes incorporating the quasimolecular opacities to obtain a consistent set of temperatures for both the variables and nonvariables in and around the ZZ Ceti instability strip. We have found four nonvariable stars inside the instability strip. Taken at face value, this indicates that temperature is not the only parameter that determines if a star pulsates or not, but the large uncertainties in the temperature determinations makes it impossible for us to offer this as more than just a suggestion at the present time.
\end{abstract}

\section{INTRODUCTION}

The ZZ Ceti stars, or pulsating DA white dwarf stars (DAV), are the single white dwarfs with hydrogen atmosphere that show multiperiodic light variations (McGraw 1977,1979 ). These variations are due to nonradial $g$-mode pulsations (Kepler 1984, and references therein). The periods of the variations range from 109 to $1186 \mathrm{~s}$, and the fractional amplitudes from $\sim 1 \%$ to $28 \%$. These variables lie in a narrow instability strip centered near the temperature of maximum photospheric hydrogen opacity-i.e., at the extension of the Cepheid instability strip down to the white dwarf sequence (McGraw 1979). Previous studies of the colors of these stars have concluded that most and perhaps all DA white dwarfs are photometric variables in the temperature range $13200 \mathrm{~K} \gtrsim T_{\text {eff }} \gtrsim 11500 \mathrm{~K}$ (Fontaine et al. 1982; Greenstein 1982). Analyses of the ZZ Ceti instability strip using the IUE Observatory images have been conducted previously by Wesemael et al. (1986), Lamontagne et al. (1987), and Lamontagne et al. (1989). A recent analysis of the instability strip using optical line profiles from $\mathrm{H} 8$ to $\mathrm{H} \gamma$ has been presented by Daou et al. (1990). Dolez et al. (1991), also studying high signal-to-noise optical spectra, found a few nonpulsating DA white dwarfs inside the ZZ Ceti instability strip.

The evidence suggests that except for their photometric variability the DAVs are otherwise normal white dwarfs, and that it is likely that they are broadly representative of all DA white dwarfs-i.e., structurally similar to some $80 \%$ of the known white dwarfs (Fontaine \& Wesemael 1987). If all the stars in the instability strip were found to be variable, then observationally it would be temperature alone that would determine which stars are pulsationally unstable. Further, we could conclude that all the stars have similar total masses and hydrogen-layer masses, as the blue and red edges of the instability strip are functions of stellar mass and the mass of the surface hydrogen layers. For a recent review of the pulsating white dwarfs, see Kawaler \& Hansen (1989).

Because the ZZ Ceti stars are relatively cool and therefore old $\left(\tau_{\text {age }} \simeq 5 \times 10^{8} \mathrm{yr}\right.$, Wood 1990), and the time scale for gravitational settling is very short compared to their lifetimes, all the hydrogen should be at the surface of the star (Schatzman 1958; Pelletier et al. 1989), unless convective mixing with the subsurface helium layer has already taken place. This convective mixing changes the atmospheric abundances at temperatures lower than 11500 $\mathrm{K}$ for stars with $\mathrm{H}$-layer smaller than $10^{-11} \mathscr{M}_{\odot}$ (see Fontaine \& Wesemael 1991 for a review).

To determine the boundaries and purity of the instability strip, we analyzed all the archival IUE images for all the DA stars with optical colors close to the ZZ Ceti instability strip, plus all DA4 and DA5 stars reported in Wegner \& Swanson (1991) as having IUE images. The analysis was made with a model atmosphere grid including the quasimolecular opacities responsible for the identified features at 1400 and $1600 \AA$ in the IUE spectra of DA white dwarfs (Koester et al. 1985; Nelan \& Wegner 1985).

In the remaining paragraphs, we will discuss our temperature estimates, the accuracy of the individual effective temperature determination, the determination of the blue and red edges of the instability strip, and the statistics of the purity of the instability strip.

\section{IUE IMAGES}

All the IUE images were obtained from the archives of the Canadian Astronomical Data Center at DAO, and we used all the SWP, LWP, and LWR images available, together with Strömgren photometry obtained by Wegner (1979, 1983), and Fontaine et al. (1985). A list of the images available for each star can be found in Wegner \& Swanson (1991). For the stars with no published Strömgren photometry, we used the Johnson photometry re- 
ported in McCook \& Sion (1987). We use the standard IUE calibration (Bohlin \& Holm 1980), the correction derived by Hackney et al. (1982) to account for wavelength- and exposure-dependent continuum distortions, and the absolute flux recalibration of the IUE cameras by Bohlin et al. (1990) for the SWP and LWR images, and Oliversen (1988) for the LWP images. We also corrected the fluxes for the sensitivity degradation of the cameras with time, as described by Bohlin \& Grillmair (1988) and Teyas \& Garhart (1990). Next, we averaged the spectra into $20 \AA$ bins for the SWP camera, and $30 \AA$ bins for the LWP and LWR camera, to give a less noisy continuum with adequate spectral resolution in the region where the quasimolecular features are important. Finally, we transformed the Strömgren and Johnson photometry to fluxes using the calibration of Heber et al. (1984).

\section{MODEL ATMOSPHERES}

The grid of model atmospheres we used is new, and similar to the one reported in Nelan \& Wegner (1985). It ranges from $8000 \mathrm{~K} \leqslant T_{\text {eff }} \leqslant 18000 \mathrm{~K}$, with $500 \mathrm{~K}$ steps, and $7.4 \leqslant \log g \leqslant 8.6$, with 0.6 dex steps. The models have pure hydrogen composition, and include the features at 1400 and $1600 \AA$ detected in the IUE spectra of several cool DA white dwarfs, and identified by Koester et al. (1985) and Nelan \& Wegner (1985) as Ly $\alpha$ satellite absorption from the $\mathrm{H}_{2}^{+}$quasimolecule and as a resonance broadening of $\mathrm{Ly}$ $\alpha$ due to the $\mathrm{H}_{2}$ quasimolecule, respectively. See Wesemael et al. (1986) for a comparison of the results obtained with the models by Nelan and Wegner (1985) and Koester et al. (1985), as well as a comparison with the temperatures derived from optical photometry and spectrophotometry.

For stars hotter than $18000 \mathrm{~K}$, we used the grid of models of Wesemael et al. (1980).

\section{EFFECTIVE TEMPERATURES}

We derived effective temperatures by fitting the observed spectra with the new grid by least-squares using the Levenberg-Marquardt method (Press et al. 1986). Each pixel was weighted by the inverse of its variance (weight $=1 / \sigma^{2}$ ), and we excluded the pixels blueward of $1250 \AA$ to prevent contamination by the geocoronal Ly $\alpha$ line; we also excluded saturated pixels. We forced the observed fluxes to match the models at the reddest bin, usually the " $y$ " Strömgren magnitude at $5500 \AA$, for normalization. The computer program used in the analysis was developed by Robert Lamontagne, from the Université de Montréal. We assumed $\log g=8.0$ - the observed mean gravity for DA white dwarfs - for all the fits (Shipman 1979; Koester et al. 1979; Bergeron et al. 1991). For example, Bergeron et al. (1991) quote $\langle\log g\rangle=7.85 \pm 0.24$. Note that although a change of $\Delta \log g=0.3$ introduces a change in $T_{\text {eff }}$ smaller than $100 \mathrm{~K}$-i.e., smaller than the average internal dispersion of the fit-future work of this kind should use a grid with smaller steps in $\log g$, and the approximation of con- stant $\log g$ should be lifted, even though it will introduce an additional free parameter to the fit.

We derived two sets of temperature estimates. In the first set we use all the IUE and photometric data available; in the second we use only the SWP image, and force the theoretical models to match the observations at $1950 \AA$ for normalization. Table 1 lists the results of both techniques. The effective temperatures derived from the SWP image alone (column 5) are hotter than the temperatures derived from all available data (column 3 ) by an average of +743 $\pm 563 \mathrm{~K}$, and this systematic difference indicates that the external error on the temperature determination is much larger than the internal error of the fit (columns 4 and 6). These external errors probably result from one or a combination of the following: the use of different normalization wavelengths for the two methods ( $5500 \AA$ for all data, and $1950 \AA$ for the SWP fit), possible mismatches of the optical and ultraviolet data, or errors in the flux calibrations.

\section{TIME SERIES PHOTOMETRY}

Because beating between pulsation modes can reduce the photometric variations of a real $\mathrm{ZZ}$ Ceti star to undetectable levels for up to $\sim 60 \mathrm{~min}$ (Hesser et al. 1976; McGraw 1977; Kepler et al. 1983), in determining whether a star is variable or not (column 2 of Table 1) we obtain at least 3 consecutive hours of time series photometry data with a time resolution of typically $10 \mathrm{~s}$. In addition to the beating down of the light curve amplitude, we observe DAVs with amplitudes as small as $0.6 \%(\simeq 6$ $\mathrm{mmag}$ ), and the $3 \mathrm{~h}$ runs are needed to give detection limits a factor two or more smaller than this. In Table 2 we present the limits for the nonvariable stars we have observed.

In our program of time series photometry we have also found a new variable star, WD 1236-495 (Kanaan et al. 1992). Most of these observations were obtained with a 2-star photometer (target and comparison star, Nather 1973 ) on the $1.6 \mathrm{~m}$ telescope of Laboratório Nacional de Astrofísica (LNA), in Brazil.

The other nonvariable stars listed in Table 1 are from the observations of Robinson \& McGraw (1976), McGraw (1979), Lawrence et al. (1967), Hesser, et al. (1969), Hesser \& Lasker (1971, 1972), Richer \& Ulrych (1974), and Dolez et al. (1991). Note, however, that these other searches for new variables did not, in general, obtain $3 \mathrm{~h}$ runs; most runs did not have a detection limit lower than $0.6 \%$, and the sample is known to be contaminated by variable stars (Kepler et al. 1983; Kanaan et al. 1992).

\section{INSTABILITY STRIP}

The first obvious conclusion of the analysis of Table 1 is that there is indeed a well-defined instability strip, i.e., a region of effective temperatures in which most stars are variable. Also, we find that the observed extent and limits of the strip depends systematically on the data used in obtaining the temperature estimates. Specifically, if we adopt the temperatures estimated by fitting to all the data 
TABLE 1. Best-fit effective temperatures.

\begin{tabular}{|c|c|c|c|c|c|c|}
\hline Star & Variable & $T_{\text {eff }}$ & $\sigma_{T_{\text {eff }}}$ & $T_{\mathrm{eff}}^{\mathrm{SWP}}$ & $\sigma_{T_{\mathrm{eff}}}$ & Alias \\
\hline WD $1108+325$ & & 39100 & 230 & & & Ton 60 \\
\hline WD $1033+464$ & & 25900 & 93 & & & GD 123 \\
\hline WD $1247+553$ & & 21500 & 240 & 21700 & 310 & PG, GD319 \\
\hline WD $1026+002$ & & 18200 & 400 & 19900 & 390 & $\mathrm{PG}^{1}$ \\
\hline WD $0413-077$ & NV & 16800 & 220 & 19400 & 320 & 40 Eri B \\
\hline WD $1143+321$ & & 16020 & 54 & 17000 & 110 & G148-7 \\
\hline WD $1105-048$ & & 15870 & 35 & 16720 & 68 & G163-50 \\
\hline WD $0406+169$ & & 15740 & 89 & 16100 & 150 & LP414-101 \\
\hline WD $2126+734$ & NV & 15440 & 92 & 16800 & 140 & G261-43 \\
\hline WD $1311+129$ & & & & 15400 & 180 & $\mathrm{PG}^{2}$ \\
\hline WD $2007-303$ & & 15080 & 41 & 16390 & 93 & LTT7987 \\
\hline WD $1919+145$ & & 15080 & 45 & 15700 & 230 & GD 219 \\
\hline WD $1327-083$ & NV & 14670 & 24 & 15520 & 69 & W485A \\
\hline WD $2047+372$ & & 14660 & 54 & 15100 & 210 & G210-26 \\
\hline WD $0352+096$ & NV & 14420 & 32 & 15000 & 150 & HZ4 \\
\hline WD $0148+467$ & & 14130 & 35 & 14800 & 120 & GD279 \\
\hline WD $0943+441$ & NV & 13790 & 79 & 15080 & 38 & SA29-130 \\
\hline WD $1213+528$ & NV & & & $13500^{1}$ & 120 & Case $1^{1}$ \\
\hline WD $0713+584$ & & & & $13400^{1}$ & 29 & GD294 \\
\hline WD $0231-054$ & NV & 12840 & 25 & 12910 & 22 & GD31 \\
\hline WD $2341+322$ & NV & 12490 & 27 & 13170 & 18 & $\mathrm{G} 130-5^{3}$ \\
\hline WD $1425-811$ & V & 12230 & 44 & 12640 & 39 & L19-2 \\
\hline WD $0401+250$ & NV & 12180 & 27 & 12870 & 33 & G8-8 \\
\hline WD $1647+591$ & V & 12120 & 11 & 12260 & 15 & G226-29 \\
\hline WD $1053-550$ & NV & & & $12000^{1}$ & 520 & BPM20383 \\
\hline WD $1855+338$ & V & 12040 & 90 & 12060 & 18 & G207-9 \\
\hline WD $1935+276$ & V & 11970 & 91 & 12370 & 31 & G185-2 \\
\hline WD $0921+354$ & V & 11840 & 91 & 13150 & 56 & G117-B15A \\
\hline WD $0133-116$ & V & 11830 & 75 & 12400 & 130 & R548 \\
\hline WD $1022+050$ & NV & 11700 & 40 & 13350 & 62 & PG,LP550-52 \\
\hline WD $2326+049$ & V & 11380 & 30 & 11780 & 41 & G29-38 \\
\hline WD $1236-495$ & V & 11200 & 55 & 11560 & 52 & BPM $37093^{4}$ \\
\hline WD $0858+363$ & $\mathbf{V}$ & 11100 & 60 & 12000 & 55 & GD 99 \\
\hline WD $0255-705$ & NV & 11010 & 38 & 12000 & 160 & BPM2819 \\
\hline WD $1559+369$ & $\mathbf{V}$ & 10880 & 30 & 11560 & 41 & R808 \\
\hline WD $1307+354$ & $\mathbf{V}$ & 10300 & 110 & 11740 & 62 & GD154 \\
\hline WD $2105-820$ & NV & 10300 & 44 & 10020 & 21 & BPM1266 \\
\hline WD $2246+223$ & NV & 10280 & 45 & 10230 & 26 & G67-23 \\
\hline WD $1544-377$ & & 10000 & 130 & 10610 & 25 & L481-60 \\
\hline WD $0839-327$ & & 8800 & 26 & 9630 & 12 & L532-81 \\
\hline WD $2359-434$ & & 7760 & 46 & 8150 & 24 & BPM45338 \\
\hline
\end{tabular}

${ }^{1}$ Spectra and colors indicate a composite with a cool object. PG1026+002 and Case 1 are known binary stars.

${ }^{2}$ The star is classified as a DBA3 in McCook \& Sion (1987).

${ }^{3}$ G130-5 is a binary system with a $9 "$ separation, composed of a DA4 and a dM5 (Oswalt et al. 1988).

${ }^{4}$ New variable ZZ Ceti star (Kanaan et al. 1992).

TABLE 2. Limits on pulsation from time series photometry.

\begin{tabular}{cc}
\hline \hline Star & Limit (mmag) \\
\hline WD 1327-083 & 2.8 \\
WD 0352+096 & 4.5 \\
WD 1213+528 & 1.5 \\
WD 1022+050 & 1.4 \\
WD 0401+250 & 4.0 \\
WD 1053-550 & 1.2 \\
WD 0255-705 & 2.8 \\
WD 2105-820 & 2.9 \\
WD 1123+189 & 3.3 \\
\hline \hline
\end{tabular}

on the star, the instability strip spans the temperature range $12230 \geqslant T_{\text {eff }} \geqslant 10300$, with the star L19-2 defining the blue edge, and GD 154 defining the red edge. Using this temperature scale (see column 3 of Table 1), the instability strip contains known 11 variable stars (the other known 11 variables have not been observed with $I U E$ ) and 4 nonvariables: PG1022+050, G8-8, BPM20383, and BPM2819. In Sec. VIII we will discuss the accuracy of the temperatures. If instead we adopt the temperatures estimated using the SWP images only (column 5 of Table 1) the instability strip spans $13150 \geqslant T_{\text {eff }} \geqslant 11560 \mathrm{~K}$, with G117-B15A at the blue edge and R808 at the red edge. Again, there are 11 variables and 4 nonvariable stars-GD 31, G8-8, BPM20383, and BPM 2819-inside the instability strip, the latter three being common with the 


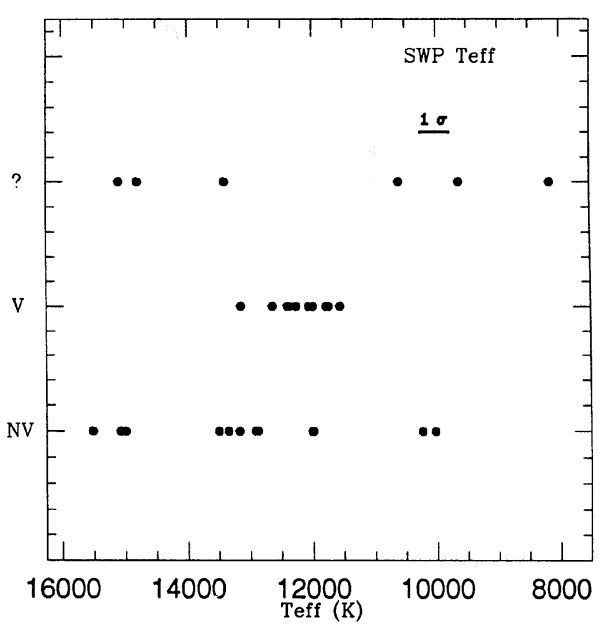

FIG. 1. Effective temperatures derived from the $I U E$ SWP images only. On the $y$ axis, the question mark designs stars that have not been looked for variability yet, $\mathrm{V}$ represents variable stars, and NV represents nonvariable stars. The $1 \sigma$ bar is the mean standard deviation for the four nonvariable stars inside the instability strip, $462 \mathrm{~K}$. The instability strip ranges $(13150 \pm 400) \geqslant T_{\text {eff }} \geqslant(11560 \pm 400) \mathrm{K}$.

nonvariable list determined using all the data. Figure 1 displays the temperatures derived from the SWP data for variable and nonvariable stars.

\section{MODELS WITH REDDENING}

To test the solutions obtained with the two different normalizations-i.e., at the red bin of the SWP camera (1950 $\AA$ ) or the Strömgren " $y$ " magnitude (or Johnson " $V$ " magnitude for WD0406+169, WD2007-303, $\mathrm{WD} 1247+553$, and WD1307+354), at $5500 \AA$-we included an extra parameter in our fit, the color excess $\mathrm{E}_{B-V}$, adopting the extinction law of Seaton (1979). The effect of a small extinction coefficient is to depress considerably the ultraviolet flux relative to the optical. For example, even an extinction coefficient of $\mathrm{E}_{B-V}=0.01$ causes a reduction in the UV flux by 5\% (Thejll et al. 1991).

With the inclusion of this ad hoc parameter, the agreement of the effective temperatures obtained with the two normalizations was better, suggesting that the reddening, or mismatching of the ultraviolet and optical fluxes, could be the main effect causing the differences in Table 1 . The mean difference between $T_{\text {eff }}$ obtained from the SWP image only and $T_{\text {eff }}$ from all the data, including the optical photometry, was then $\Delta T_{\text {eff }}=273 \pm 380 \mathrm{~K}$, consistent within 1 $\sigma$. We will use the value of $380 \mathrm{~K}$ as the external error on the temperature.

One of the possibilities for inaccuracies for the faint stars in miscentering in the $I U E$ aperture which causes the measured IUE flux to be lower than the true value. The reason we suspect miscentering is that stars fainter than $V=15.5$ (Sonneborn et al. 1987; Grady \& Taylor 1989)the minority of our sample-are too faint to be seen on the $I U E$ guide sensors and therefore had to be centered by using an offset from a nearby bright star, an operation which is sometimes less than successful (Sonneborn et al. 1987). Another problem is that, as the star is faint, the scatter in the observed fluxes is large in both the ultraviolet and optical, increasing the uncertainty of matching UV and optical fluxes. For the fainter objects (Ton 60, PG $1311+129$, and G117-B15A), miscentering of the star in the $I U E$ aperture is possible. For these faint stars the effective temperatures derived from the SWP image alone should be more accurate, because the fit is only to the shape of the spectra, and does not rely on its overall flux calibration.

\section{ACCURACY OF THE OBTAINED TEMPERATURES}

As we discussed above, we assume a surface gravity of $\log g=8.0$ for all our fits, and for stars in this temperature range, we expect a scatter of $\Delta \log g=0.3$ (Daou et al. 1990). For these objects, a change in the surface gravity of $\Delta \log g=0.3$ gives $\Delta T_{\text {eff }} \simeq 100 \mathrm{~K}$.

The main uncertainty in the absolute temperature determination comes from the $10 \%$ systematic external uncertainty in the absolute flux calibration of the IUE cameras (Bohlin et al. 1991). This $10 \%$ uncertainty in the flux results in an uncertainty of $\Delta T_{\text {eff }}=240 \mathrm{~K}$ for the stars in the temperature range of the $\mathrm{ZZ}$ Ceti stars, when using the normalization at the Strömgren " $y$ " or Johnson " $V$ " magnitudes. When we use the normalization at the red bin of the SWP camera, there is no uncertainty from this effect, since we then fit only the shape of the spectra, not its absolute calibration.

There is another uncertainty affecting our fitted temperatures, the mixing length parameter used in the models to describe the efficiency of convection. Winget et al. (1982), Fontaine et al. (1984), and Tassoul et al. (1990) demonstrate that the change of parameters from ineffective ML1 to effective ML3 changes the position of the theoretical instability strip for the ZZ Ceti stars by up to $500 \mathrm{~K}$. Bergeron et al. (1992) indicates that the derived effective temperature could change systematically by about $1000 \mathrm{~K}$ if we change from ML1 to ML3, in the temperature range of the ZZ Ceti stars. The grid of models used in this study was calculated with a mixing length $l=1.5 H_{P}$, where $H_{P}$ is the pressure scale height. We do not have at the moment grids of models calculated with different mixing length parameters, or theories, to compare its effect on the observed flux in the ultraviolet, and its consequent uncertainty in the temperatures derived here.

\section{STATISTICS OF THE NONVARIABLES}

Due to the large internal and external errors on the obtained temperatures, as well as the narrowness of the instability strip, we must estimate the significance of the result that there are nonvariable stars inside the instability strip. To estimate the significance of each of the nonvariables' temperature determinations, we first calculated

$$
\sigma_{\text {Total }}=\left[\sigma_{\text {internal }}^{2}+\sigma_{\Delta \log g}^{2}+\sigma_{\text {external }}^{2}\right]^{1 / 2},
$$


TABLE 3. Probabilities for nonvariables stars.

\begin{tabular}{lc}
\hline \multicolumn{1}{c}{ Star } & $P_{\text {out }}$ \\
\hline SA29-130 & 0.997 \\
Case 1 & 0.987 \\
GD 294 & 0.982 \\
G130-5 & 0.821 \\
G8-8 & 0.472 \\
BPM20383 & 0.395 \\
PG 1022+050 & 0.179 \\
BPM2819 & 0.128 \\
BPM1266 & 0.500 \\
G67-23 & 0.514 \\
L481-60 & 0.693 \\
\hline \hline
\end{tabular}

for all the temperatures obtained using the SWP images only. It is a conservative estimate of the total uncertainty in the temperature caused by (i) the formal uncertainty for the temperature fit (internal error, column 7 of Table 1) (ii) the uncertainty given by a possible $\Delta \log g=0.3$ change in the surface gravity $\left(\sigma_{\Delta \log g}=100 \mathrm{~K}\right)$, and (iii) the external error, which we will assume to be $\sigma_{\text {external }}=380 \mathrm{~K}$. We did not include the standard deviation due to the absolute flux calibration because it should affect only the absolute temperatures, and be included in the external error. We consider $\sigma_{\text {Total }}$ a conservative estimate because, for the temperatures derived from the SWP images only, there is no match between optical and IUE fluxes. $T_{\mathrm{eff}}^{\mathrm{SWP}}$ are the temperatures we will use in our statistical estimate of the purity of the instability strip.

We calculated the difference in temperature to the blue and red edges, including the uncertainties in the temperature of the edges themselves $\left(\sigma_{\text {Total }}^{\text {edge }}=400 \mathrm{~K}\right.$ ), and the total probability that the star would lie either to the blue or to the red of the instability strip.

For the temperature fits using the SWP images only, the probability that the nonvariable stars GD31, G8-8, BPM20383, and BPM2819 are outside the instability strip are $34.2 \%, 31.8 \%, 34.7 \%$, and $24.8 \%$, respectively. There is also a probability of $27.4 \%, 36.2 \%$, and $48.4 \%$ that Case 1, PG1022+050, and G130-5 are inside the instability strip. The compound probability that the instability strip is pure, i.e., contain only variable stars, is $2.2 \times 10^{-3}$, equivalent to outside of $2.8 \sigma$, or 1 chance in 450 .

For the temperatures derived using both the IUE images and the optical photometry, the probability that the nonvariable stars are outside the instability strip $P_{\text {out }}$ are found in Table 3.

The compound probability that the instability strip is pure, i.e., contain only variable stars, is $6.0 \times 10^{-4}$, equivalent to outside of $3.2 \sigma$.

The very simple confidence estimate derived here might be misleading as the large temperature uncertainties for each star puts every nonvariable less than two standard deviations from the boundaries of the instability strip.

\section{DISCUSSION}

The main conclusion of our analysis is that most but not all-75\% - stars inside a narrow temperature range are variable, and therefore the conclusion of Fontaine et al. (1982) that the temperature is the main parameter for determining whether a star is variable or not still holds. The temperature of the instability strip itself is uncertain by at least $273 \pm 380$, the external error on our fit.

We have, on the other hand, found four stars inside the instability strip that are not observed to be variables. They constitute $27 \%$ of the stars in the instability strip, independent of which of the two temperature scales is adopted. The scale obtained by fitting to the SWP images only has the smaller uncertainties because here the fitting is essentially to the shape of the spectra and the absolution flux calibration is unimportant. By contrast, although the scale using all the IUE images and optical photometry data is the one having the greatest leverage on the energy distribution, it is subject to uncertainties on the absolute flux calibration of the $I U E$ images through, for example, problems of centering faint stars in the $I U E$ aperture using blind offsets.

There are a few effects that must be taken in account when analyzing such statistics:

(1) Certain combinations of the indices of the spherical harmonics $l$ and $m$, when viewed from certain aspects, show no luminosity variations, because the surface brightness distribution over the observable hemisphere of the star averages out in time. For example, when the rotation axis of a variable star is seen pole on, the pulsation modes which are symmetrical about the pole cancel out, and we see no observable variations, even though the star is variable. If the modes are rotationally split, on the other hand, the effect will make modes with different $m$ values have different amplitudes, but probably not cancel out all the variations (Pesnell 1985). This factor cannot explain such large percentage of nonvariables because it requires very specific orientations (Fontaine et al. 1982).

(2) There is an observational limit on the detectable pulsational amplitude. Considering that some known pulsators have very small amplitudes $(0.6 \% \simeq 6 \mathrm{mmag}$ for G226-29, $0.4 \% \simeq 4 \mathrm{mmag}$ for BPM37093), we could claim that a pulsating star is nonvariable if its amplitude is below our detection limit.

Our conclusion is that, taken at face value, there is a significant number of nonvariable stars inside the $\mathrm{ZZ}$ Ceti instability strip. The relatively large remaining uncertainties in the temperature determinations make it impossible for us to offer this conclusion as more than just a suggestion at the present time. If these nonvariables are indeed inside the instability strip, these stars could have different hydrogen surface layer mass, and or different stellar masses. A statistical analysis with a larger number of stars, both variable and nonvariable, is necessary, and we are in the processes of observing such stars with both the IUE satellite and optical spectroscopy, as well as with timeseries photometry to improve the statistical significance of our conclusions. A study of the masses (surface gravities) and the effects of convective efficiencies on the IUE spectra should also be undertaken.

This work was partially supported by grants from 
CNPq (Brazil), NSERC (Canada), and FCAR (Canada). We thank Robert Lamontagne for allowing us to use his computer program, and Pierre Bergeron and François
Wesemael for providing us with the hot white dwarf model grid. We also thank Matt Wood for helpful discussions and suggestions.

\section{REFERENCES}

Bergeron, P., Saffer, R., \& Liebert, J. 1991, in White Dwarfs, edited by G. Vauclair and E. Sion (Kluwer, Dordrecht), p. 75

Bergeron, P., Wesemael, F. \& Fontaine, G. 1992, ApJ, 387, 288

Bohlin, R. C. 1986, ApJ, 308, 1001

Bohlin, R. C., \& Grillmair, C. J. 1988, ApJS, 66, 209

Bohlin, R. C., Harris, A. W., Holm, A. V., \& Guy, C. 1990, ApJ, 73, 413

Bohlin, R. C., \& Holm, A. V. 1980, NASA IUE Newsl., 10, 37

Bradley, P. A., \& Winget, D. E. 1991, ApJS, 75, 463

Daou, D., Wesemael, F., Bergeron, P., Fontaine, G., \& Holberg, J. B. 1990, ApJ, 364, 242

Dolez, N., Vauclair, G., \& Koester, D. 1991, in White Dwarfs, edited by G. Vauclair and E. Sion (Kluwer, Dordrecht), p. 361

Fontaine, G., Bergeron, P., Lacombe, P., Lamontagne, R., \& Talon, A. 1985, AJ, 90, 1094

Fontaine, G., McGraw, J. T., Dearborn, D. S. P., Gustafson, J., \& Lacombe, P. 1982, ApJ, 258, 651

Fontaine, G., Tassoul, M., \& Wesemael, F. 1984, in Proceedings of the 25th Liege International Astrophysical Colloquium: Theoretical Problem in Stellar Stability and Oscillations (Université de Liege, Liege), p. 328

Fontaine, G., \& Wesemael, F. 1987, in Proceedings of the IAU Colloquium 95, The Second Conference on Faint Blue Stars, edited by A. G. D. Philip, D. S. Hayes, and J. W. Liebert (Davis, Schenectady), p. 669

Fontaine, G., \& Wesemael, F. 1991, in Proceedings of the IAU Symposium 145, Evolution of Stars: The Photospheric Abundance Connection, edited by G. Michaud and A. Tutukov (Kluwer, Dordrecht), p. 421

Greenstein, J. L. 1982, ApJ, 258, 661

Grady, C. A., \& Taylor, M. A. 1989, in IUE Data Analysis Guide, IUE NASA Newsletter No. 39 , p. 7

Hackney, R. L., Hackney, K. R. H., \& Kondo, Y. 1982, in Advances in Ultraviolet Astronomy: Four Years of IUE Research, NASA CP-2238 (NASA, Washington), p. 335

Heber, U., Hunger, K., Jones, G., \& Kudritzki, R. P. 1984, A\&A, 130, 119

Hesser, J. E., \& Lasker, B. M. 1971, in IAU Symposium No. 42, p. 41

Hesser, J. E., \& Lasker, B. M., 1972, in Variable Stars, Proceedings of the IAU Colloquium 15, p. 160

Hesser, J. E., Lasker, B. M., \& Neupert, 1976, ApJ, 209, 853

Hesser, J. E., Ostriker, J. P., \& Lawrence, G. M. 1969, ApJ, 155, 919

Kanaan, A., Kepler, S. O., Giovannini, Jr., O. \& Diaz, M. 1992, ApJ, 390, L89

Kawaler, S. D., \& Hansen, C. J. 1989, in White Dwarfs, Proceedings of the IAU Colloquium 114, edited by G. Wegner (Springer, Berlin), p. 97 Kepler, S. O. 1984, ApJ, 286, 314
Kepler, S. O., Robinson, E. L., \& Nather, R. E. 1983, ApJ, 271, 744

Koester, D., Schulz, H., \& Weidemann, V. 1979, A\&A, 76, 262

Koester, D., Weidemann, V., Zeidler-K. T., E.-M., \& Vauclair, G. 1985, A\&A, 142, L5

Lamontagne, R., Wesemael, F., Fontaine, G., Wegner, G., \& Nelan, E. P. 1987, in The Second Conference on Faint Blue Stars, Proceedings of the IAU Colloquium 95, edited by A. G. D. Philip, D. S. Hayes, and J. W. Liebert (Davis, Schenectady), p. 677

Lamontagne, R., Wesemael, F., \& Fontaine, G. 1989, in White Dwarfs, Proceedings of the IAU Colloquium 114, edited by G. Wegner (Springer, Berlin), p. 240

Laker, B. M., \& Hesser, J. E. 1971, ApJ, 163, L89

Lawrence, J. M., Ostriker, J. P., \& Hesser, J. E. 1967, ApJ, 148, L161

McCook, G. P., Sion, E. M. 1987, ApJS, 65, 603

McGraw, J. T. 1977, ApJ, 214, L123

McGraw, J. T. 1979, Ph.D. thesis, The University of Texas at Austin

Nather, R. E. 1973, Vistas in Astronomy, 15, 91

Nelan, E. P., \& Wegner, G. 1985, ApJ, 289, L31

Oliversen, N. A. 1988, IUE Newsletter, 35, 55

Oswalt, T. D., Hintzen, P. M., \& Luyten, W. J. 1988, ApJS, 66, 391

Pelletier, C., Fontaine, G., \& Wesemael, F. 1989, in White Dwarfs, Proceedings of the IAU Colloquium 114, edited by G. Wegner (Springer, Berlin), p. 249

Pesnell, W. D. 1985, ApJ, 292, 238

Press, W. H., Flannery, B. P., Teukolsky, S. A., \& Vetterling, W. T. 1986, Numerical Recipes (Cambridge University Press, Cambridge)

Richer, H. B., \& Ulrych, T. J. 1974, ApJ, 192, 719

Robinson, E. L., \& McGraw, J. T. 1976, ApJ, 207, L37

Schatzman, E. 1958, White Dwarfs (North Holland, Amsterdam)

Seaton, M. J. 1979, MNRAS, 187, 73P

Shipman, H. 1979, ApJ, 228, 240

Sonneborn, G., Oliversen, N. A., Imhoff, C. L., Pitts, R. E., \& Holm, A V. 1987, in IUE Observing Guide, IUE NASA Newsletter No. 32, p. 20

Tassoul, M., Fontaine, G., \& Winget, D. E. 1990, ApJS, 72, 335

Teyas, T. J., \& Garhart, M. P. 1990, IUE Newsletter, 41, 94

Thejll, P., Vennes, S., \& Shipman, H. L. 1991, ApJ, 370, 355

Wegner, G. 1979, AJ, 84, 1384

Wegner, G. 1983, AJ, 88, 109

Wegner, G., \& Swanson, S. R. 1991, ApJS, 75, 507

Wesemael, F., Auer, L. H., Van Horn, H. M., \& Savedoff, M. P. 1980, ApJS, 43, 159

Wesemael, F., Lamontagne, R., \& Fontaine, G. 1986, AJ, 91, 1376

Winget, D. E., Van Horn, H. M., Tassoul, M., Hansen, C. J., Fontaine, G., \& Carrol, B. W. 1982, ApJ, 252, L65

Wood, M. A. 1990, JRASC, 84, 150 